\title{
Risk factors, comorbidities, quality of life, and complications after surgery in idiopathic normal pressure hydrocephalus: review of the INPH-CRasH study
}

\author{
Hanna Israelsson, MD, PhD, ${ }^{1,2}$ Jenny Larsson, MD, ${ }^{1}$ Anders Eklund, $\mathrm{PhD},{ }^{3}$ and Jan Malm, MD, PhD' \\ ${ }^{1}$ Department of Clinical Science, Neurosciences, Umeå University, Umeå; '2Department of Health, Medicine and Caring Sciences \\ (HMV), Linköping University Hospital, Linköping; and 'Department of Radiation Sciences, Umeå University, Umeå, Sweden
}

OBJECTIVE Idiopathic normal pressure hydrocephalus (INPH) is a dementia treatable by insertion of a shunt that drains CSF. The cause of the disease is unknown, but a vascular pathway has been suggested. The INPH-CRasH (Comorbidities and Risk Factors Associated with Hydrocephalus) study was a modern epidemiological case-control study designed to prospectively assess parameters regarding comorbidities and vascular risk factors (VRFs) for INPH, quality of life (QOL), and adverse events in patients with shunted INPH. The objective of this review was to summarize the findings of the INPH-CRasH study.

METHODS VRFs, comorbidities, QOL, and adverse events were analyzed in consecutive patients with INPH who underwent shunt placement between 2008 and 2010 in 5 of 6 neurosurgical centers in Sweden. Patients $(n=176$, within the age span of 60-85 years and not having dementia) were compared to population-based age- and gender-matched controls ( $n=368$, same inclusion criteria as for the patients with INPH). Assessed parameters were as follows: hypertension; diabetes; obesity; hyperlipidemia; psychosocial factors (stress and depression); smoking status; alcohol intake; physical activity; dietary pattern; cerebrovascular, cardiovascular, or peripheral vascular disease; epilepsy; abdominal pain; headache; and clinical parameters before and after surgery. Parameters were assessed through questionnaires, clinical examinations, measurements, ECG studies, and blood samples.

RESULTS Four VRFs were independently associated with INPH: hyperlipidemia, diabetes, obesity, and psychosocial factors. Physical inactivity and hypertension were also associated with INPH, although not independently from the other risk factors. The population attributable risk percent for a model containing all of the VRFs associated with INPH was $24 \%$. Depression was overrepresented in patients with INPH treated with shunts compared to the controls ( $46 \%$ vs $13 \%$, $p<0.001)$ and the main predictor for low QOL was a coexisting depression $(p<0.001)$. Shunting improved QOL on a long-term basis. Epilepsy, headache, and abdominal pain remained common for a mean follow-up time of 21 months in INPH patients who received shunts.

CONCLUSIONS The results of the INPH-CRasH study are consistent with a vascular pathophysiological component of INPH. In clinical care and research, a complete risk factor analysis as well as screening for depression and a measurement for QOL should probably be included in the workup of patients with INPH. The effect of targeted interventions against modifiable VRFs and antidepressant treatment in INPH patients should be evaluated. Seizures, headache, and abdominal pain should be inquired about at postoperative follow-up examinations.

https://thejns.org/doi/abs/10.3171/2020.7.FOCUS20466

KEYWORDS normal pressure hydrocephalus; vascular disease; vascular risk factors; outcome of surgery; adverse events; quality of life

$\mathrm{I}$ DIOPATHIC normal pressure hydrocephalus (INPH) is a dementia treatable by insertion of a CSF shunt. The disease occurs in the elderly, who are prone to several coexisting diseases and conditions. In this review, we summarize the findings of the INPH-CRasH (Comorbidities and Risk Factors Associated with Hydrocephalus) study. This was a large and prospective Swedish case-control study epidemiologically designed to address knowledge gaps regarding comorbidities (vascular comorbidity including vascular risk factors [VRFs] and manifest vascular

ABBREVIATIONS AE = adverse event; $\mathrm{AED}=$ antiepileptic drug; CRasH = Comorbidities and Risk Factors Associated with Hydrocephalus; CVD = cerebrovascular disease; EQ-VAS = EuroQol visual analog scale; EQ-5D-5L = EuroQol 5-dimensions 5-level; GDS-15 = Geriatric Depression Scale-15; INPH = idiopathic normal pressure hydrocephalus; $\mathrm{QOL}=$ quality of life; VRF = vascular risk factor; WML = white matter lesion.

SUBMITTED June 1, 2020. ACCEPTED July 27, 2020.

INCLUDE WHEN CITING DOI: 10.3171/2020.7.FOCUS20466. 
disease as well as depression), quality of life (QOL), and adverse events (AEs) after surgery in patients with INPH treated with shunts. So far, results from the INPH-CrasH study have been published in 4 papers $^{1-4}$ and 1 dissertation, ${ }^{5}$ and this review will summarize the key findings up to this point.

\section{Treatment of INPH}

INPH is a disease occurring in the elderly population. ${ }^{6,7}$ The mean age at onset is approximately 70 years. ${ }^{6,7}$ The symptomatology is often described as a triad. The first and most prominent symptom is a gradually developing symmetrical gait and balance disturbance. ${ }^{6}$ The second typical symptom is cognitive decline, which may vary from very subtle changes to manifest dementia. The third symptom of INPH is urinary problems. ${ }^{6}$ The symptoms of INPH become aggravated over time and early diagnosis and treatment is important. ${ }^{8}$ Treatment is provided by insertion of a shunt that drains CSF from the ventricles. A ventriculoperitoneal shunt is the most commonly used system. ${ }^{7}$ Another type of shunt is the lumboperitoneal shunt, which is most frequently used in Japan. ${ }^{9}$

Treatment with a shunt has been reported to improve the gait in approximately $60 \%-80 \%$ of these patients. ${ }^{10-12}$ Improvements in cognition are often somewhat less. ${ }^{13,14}$ However, in an unselected multicenter study, the total improvement rate was 39\% according to the modified Rankin Scale and $58 \%$ on a modified INPH scale,${ }^{15}$ indicating that improvement rates are probably lower in clinical reality than previously thought. There is extensive literature focusing on severe AEs after shunt surgery for INPH. A less serious but commonly occurring problem is headache caused by overdrainage. A recent study reported an $8 \%$ prevalence of postural headache. ${ }^{16}$ However, minor AEs such as headache in general (not postural), abdominal pain, and seizures or epilepsy have rarely been studied in patients with INPH.

\section{Comorbidities}

The cause of INPH is unknown but is most likely multifactorial. Assessment and treatment of comorbidities has been suggested to improve the prognosis. ${ }^{6,7,17}$ Cerebrovascular disease (CVD) is one of the most important comorbidities in INPH, ${ }^{17}$ and it has been hypothesized that vascular disease may be involved in the pathogenesis of INPH. ${ }^{18-21}$ Vascular disease is normally preceded by a large number of VRFs. ${ }^{22}$ When the INPH-CRasH study was initiated, very few studies regarding VRFs in INPH were found in the literature. ${ }^{23-26}$ In these studies, hypertension was considered the single most important VRF in INPH, and there were some indications that high cholesterol levels, diabetes, and ischemic heart disease ${ }^{25,26}$ might be overrepresented.

Depression is one of the most common and underdiagnosed comorbidities in the general elderly population. It is both common among elderly people with dementia as well as a significant risk factor for dementia. ${ }^{27,28}$ However, few studies that focus on the cognitive aspects of INPH assess the prevalence of a potentially coexisting depression.

This review aims to summarize the findings of the
INPH-CRasH study regarding the complete VRF profile, depression, QOL, and minor AEs caused by the CSF shunt. In addition, each section of the review provides a short summary of the current literature.

\section{Methods Subjects}

In this review, the Methods section has been summarized. For a detailed description and specific methodological concerns, see the original papers and the thesis. ${ }^{1-5}$ The INPH-CRasH study is registered with the ClinicalTrials. gov database (https://clinicaltrials.gov), and its registration number is NCT01850914.

The methodological approach for the INPH-CRasH study was a case-control study. Consecutive patients with INPH selected for shunt placement between 2008 and 2010 at 5 of 6 neurosurgical centers in Sweden, and registered in the Swedish Hydrocephalus Quality Register (SHQR), were included. These patients were compared with population-based gender- and age-matched controls. A total of 396 INPH patients were registered in the SHQR between 2008 and 2010. The exclusion criteria were age $<60$ or $>85$ years at the date of surgery, a known preoperative Mini-Mental State Examination score $<23$, or death. After exclusion, 239 patients were contacted for participation, and 176 chose to participate in the study (mean age $[ \pm$ SD] $74 \pm 6$ years; female patients $42 \%$ [n = 73]). Six hundred eighty-four population-based controls were enrolled, and after exclusion 368 controls chose to participate in the study (mean age $[ \pm \mathrm{SD}] 73 \pm 6$ years; female patients $37 \%$ [ $=135])$.

\section{Parameters}

Parameters were prospectively assessed through questionnaires, clinical examinations, anthropometrical measurements, ECG studies, and blood samples, as well as through several registries for both cases and controls. Investigated parameters included the following:

- The 10 VRFs that have been proven to account for approximately $90 \%$ of the risk for myocardial infarction or stroke worldwide. ${ }^{29,30}$

- Cardiovascular, cerebrovascular, and peripheral vascular disease.

- Depression, assessed by the Geriatric Depression Scale-15 (GDS-15). ${ }^{31}$

- Epilepsy.

- QOL measured by the EuroQol 5-dimensions 5-level (EQ-5D-5L) instrument. ${ }^{32}$

- Medication for vascular disease, VRFs, psychiatric symptoms, and epilepsy.

- Falls and fear of falling, measured by the Swedish Falls Efficacy Scale (FES[S]). ${ }^{33}$

- Independence measured by housing status and the need for in-home care.

In addition, clinical symptoms before and after surgery regarding gait, modified Rankin Scale score, and MiniMental State Examination score were assessed for the INPH patients, as were minor AEs after surgery (epilepsy, abdominal pain, headache). The mean follow-up time after 
surgery was 21 months (range 6-45 months). All controls were given a fictive date of surgery to enable comparisons of registry data between INPH patients and controls before and after surgery regarding AEs.

\section{Results and Discussion}

A summary of the most important results follows:

- Vascular disease and VRFs are overrepresented among the INPH patients compared with the population. ${ }^{1,5}$

- Among the patients with shunt-treated INPH, 48\% presented with symptoms of a coexisting depression or high burden of stress. ${ }^{2,5}$

- Shunting improved the patient's QOL on both a shortand long-term basis. ${ }^{3,5}$

- Epilepsy, headache, and abdominal pain remain common among patients with shunt-treated INPH at a follow-up period of 45 months. ${ }^{4}$

- INPH patients fall more often than controls and have a high level of fear of falling (conference paper ${ }^{34}$ and our unpublished data).

\section{VRFs and Vascular Disease}

VRFs and INPH

An association between VRFs and INPH has previously been suggested. ${ }^{17-21,35}$ In the INPH-CRasH study, modern VRFs were assessed with an appropriate epidemiological design and representative population-based controls. Four VRFs were independently associated with INPH (odds ratios [ORs] from the model that included all VRFs independently associated with INPH): hyperlipidemia (OR 2.4, 95\% CI 1.4-4.0), diabetes (OR 2.2,95\% CI 1.2-3.9), obesity (OR 5.4, 95\% CI 2.5-11.8), and psychosocial factors (OR 5.3, 95\% CI 3.2-8.9). For ORs for each separate risk factor, adjusted for age and gender, see Table 1. Physical inactivity and hypertension were also associated with INPH, although not independently from the other risk factors. The population attributable risk percent for a model containing all of the VRFs associated with INPH was 24\% (95\% CI 20\%-28\%), meaning that hyperlipidemia, diabetes, obesity, psychosocial factors, physical inactivity, and hypertension accounted for $24 \%$ of the INPH cases in the elderly population. These results expand and confirm the findings from previously conducted studies. ${ }^{23-26}$ In addition, the results correspond well to VRF studies in other dementias. 27,36

Since the INPH-CRasH study was initiated, some more case-control studies have been conducted, most of them with similar results. ${ }^{37-39}$ One study retrospectively investigated VRFs in a sample of 26 patients with ventriculomegaly and clinical findings of INPH without diagnosis, ${ }^{38}$ and found that hypertension was associated with these characteristics, although this finding was not significant in multivariate analyses. Another study investigated 440 patients with $\mathrm{INPH}^{37}$ and found that hypertension and diabetes were overrepresented in INPH. Both of these studies are in agreement with the INPH-CRasH study. A third study investigated a sample of 29 INPH patients without $\mathrm{CVD}^{40}$ and found no association between INPH patients and VRFs, except for a history of alcohol use disorder, in contrast to the INPH-CRasH study. A possible reason for this may be that patients with CVD were excluded. Because most patients with INPH present with signs of CVD, these patients probably represent a smaller subgroup of those with INPH. A recent review also highlights diabetes as a common comorbidity in INPH..$^{39}$

Nine years ago, the American Heart Association and American Stroke Association emphasized the importance of vascular disease and VRFs for cognitive impairment and dementia. ${ }^{36} \mathrm{~A}$ recent review that assessed the major modifiable risk factors for dementia identified these same 6 risk factors as well as smoking as the most important modifiable VRFs for dementia prevention. ${ }^{41}$

\section{Vascular Disease in INPH: Cause or Comorbidity?}

The few studies that investigate how coexisting VRFs affect the outcome of surgery in patients with INPH present conflicting results,,$^{10,21,42}$ and an association between less improvement after shunting and VRFs has not been found. However, VRFs inevitably lead to the classic vascular diseases (i.e., stroke, myocardial infarction, or peripheral vascular disease), and INPH patients presenting with such diseases are less likely to improve after surgery. ${ }^{21,43-45}$ In the INPH-CRasH study, CVD (25\% for patients vs $13 \%$ for controls, OR 2.1, 95\% CI 1.3-3.5; $\mathrm{p}=0.001$ ) and peripheral vascular disease (90\% for patients vs $75 \%$ for controls, OR 2.8, 95\% CI 1.5-5.2; $\mathrm{p}=0.001$ ), but not ischemic heart disease, were overrepresented in the INPH patients compared with the controls. Regarding ischemic heart disease, another study investigated 440 patients with INPH ${ }^{37}$ and found that cardiovascular disease was overrepresented in patients with INPH compared to the general population. However, in this study the VRFs were assessed in different ways for cases and controls.

Few studies have investigated how VRFs affect the outcome of surgery in patients with INPH, ${ }^{10,21,42}$ and an association between less improvement after shunting and VRFs has not been found. Most studies that investigate the impact of vascular disease on outcome of shunt surgery show that patients with CVD are less likely to improve after surgery:21,43-46 however, a recent study that included 979 patients suggests that vascular comorbidity may have very small effects on long-term outcome. ${ }^{47}$ Nevertheless, vascular disease is the most common cause of death in INPH patients, $11,45,48,49$ and a number of studies report CVD to be overrepresented in INPH, particularly typical signs of small-vessel disease such as white matter lesions (WMLs) and microbleeds. ${ }^{17,18,21,50,51}$ Subcortical vascular dementia is perhaps the most important differential diagnosis to $\mathrm{INPH}^{17,52}$ and is characterized by extensive, progressive WMLs as well as enlarged ventricles and hydrocephalic symptoms. ${ }^{17}$ Both postmortem studies and biopsies taken during surgery demonstrate similarities between patients with INPH and patients with subcortical vascular dementia. ${ }^{18,53}$ In recent years, some differences between the biomarker profile between INPH and subcortical ischemic vascular disease have been suggested. ${ }^{54}$

It remains unclear whether risk factors and subsequent vascular disease merely coexist with INPH or have a significant role in the pathogenesis and progression of the disease. ${ }^{17}$ It has been hypothesized that INPH and ischemic CVD share some of the same pathophysiological 
TABLE 1. The total VRF profile of patients with INPH and controls

\begin{tabular}{|c|c|c|c|c|}
\hline \multirow[b]{2}{*}{ Variable } & \multicolumn{2}{|c|}{ Frequency for Each Variable (no. with VRF/total no. reporting) } & \multicolumn{2}{|c|}{ Analyses } \\
\hline & INPH & Controls & OR $(95 \% \mathrm{Cl})$ & p Value \\
\hline Hypertension & $83 \%(131 / 157)$ & $75 \%(263 / 352)$ & $1.7(1.0-2.7)$ & 0.04 \\
\hline Hyperlipidemia & $37 \%(52 / 140)$ & $19 \%(63 / 325)$ & $2.5(1.6-3.9)$ & $<0.001$ \\
\hline Diabetes & $27 \%(38 / 142)$ & $13 \%(44 / 337)$ & $2.4(1.5-4.0)$ & $<0.001$ \\
\hline Abdominal obesity & $91 \%(128 / 140)$ & $73 \%(236 / 325)$ & $5.7(2.9-11.4)$ & $<0.001$ \\
\hline Smoking & $55 \%(95 / 172)$ & $52 \%(190 / 365)$ & $1.2(0.8-1.8)$ & 0.29 \\
\hline \multicolumn{5}{|l|}{ Physical activity } \\
\hline Active & $33 \%(53 / 160)$ & $51 \%(186 / 364)$ & $0.5(0.3-0.7)$ & $<0.001$ \\
\hline Inactive & $24 \%(40 / 166)$ & $10 \%(35 / 367)$ & $2.8(1.7-4.7)$ & $<0.001$ \\
\hline Psychosocial factors & $48 \%(83 / 172)$ & $16 \%(57 / 365)$ & $4.9(3.2-7.5)$ & $<0.001$ \\
\hline \multicolumn{5}{|l|}{ Cardiac disease } \\
\hline Ischemic & $23 \%(32 / 138)$ & $21 \%(70 / 333)$ & $1.1(0.7-1.8)$ & 0.65 \\
\hline All & $46 \%(65 / 143)$ & $41 \%(140 / 341)$ & $1.2(0.8-1.8)$ & 0.44 \\
\hline \multicolumn{5}{|l|}{ Alcohol intake } \\
\hline Moderate & $54 \%(92 / 170)$ & $67 \%(246 / 365)$ & $0.6(0.4-0.9)$ & 0.01 \\
\hline High & $20 \%(34 / 170)$ & $23 \%(83 / 365)$ & $0.9(0.6-1.5)$ & 0.77 \\
\hline \multicolumn{5}{|l|}{ Dietary pattern } \\
\hline Prudent & $74 \%(128 / 173)$ & $75 \%(276 / 367)$ & $0.9(0.6-1.4)$ & 0.74 \\
\hline Unhealthy & $18 \%(31 / 173)$ & $16 \%(60 / 366)$ & $1.1(0.7-1.8)$ & 0.74 \\
\hline
\end{tabular}

Odds ratios are for each separate VRF. All ORs were adjusted for age and gender.

mechanisms. ${ }^{18-21,35}$ WMLs are associated with more severe symptoms in INPH..$^{21,43-45}$ Patients with INPH have been proven to have a reduced cerebral blood flow in both the periventricular and frontal subcortical areas of the brain. ${ }^{55-57}$ Because the disturbances in gait and cognition are thought to arise from disturbances in the periventricular/subcortical/frontal areas of the brain, ${ }^{7}$ this links the symptomatology to possible vascular changes. The INPHCRasH study provides additional support for that theory and suggests a possible causal relationship between INPH and VRFs/vascular disease.

\section{Suggestions for the Future}

In most neurosurgical centers, when a patient with INPH is selected for surgery, no VRF analysis is performed, and existing risk factors are not specifically addressed and treated. In clinical care, the treatable VRFs should probably be screened for as part of the preoperative evaluation of INPH patients. Existing VRFs should be optimized in addition to surgery, according to evidence from modern guidelines for vascular disease and risk factors. $^{22,36}$

\section{Depression}

\section{Symptoms of Depression Are Common in INPH Patients}

Depression both severely affects cognitive function among the elderly ${ }^{27}$ and is an important risk factor for dementia. ${ }^{27,41}$ In the INPH-CRasH study, shunt-treated patients were more likely to experience both depression (defined as a GDS-15 score $\geq 5$ points: $46 \%$ for patients vs $13 \%$ for controls, OR $6.4,95 \%$ CI 3.8-10.9; p < 0.001) and severe depression (defined as a GDS-15 score $\geq 12$ points: OR 14.4, 95\% CI 3.0-68.6; $\mathrm{p}=0.001$ ) than the controls. More patients had depression before than after surgery $(56 \%$ vs $46 \%$; $\mathrm{p}=0.04)$. INPH patients also had a higher mean depression score than controls both before and after surgery - for the patients the mean scores $( \pm$ SD) were $5.9 \pm 3.8$ and $4.9 \pm 3.7$ before and after surgery, respectively, whereas for controls the mean score was $1.9 \pm$ 2.3. The OR after surgery when comparing patients and controls was 1.4 (95\% CI 1.3-1.6; $\mathrm{p}=0.001)$; before surgery the OR was 1.6 (95\% CI 1.4-1.8; $\mathrm{p}=0.001$; Fig. 1). The frequency of control volunteers with depression was in accordance with the frequency of depression in other population-based studies (up to $16 \%$ ) $^{27,58}$ and in agreement with a recent study investigating the history of psychiatric disorders in patients with INPH. ${ }^{40}$ However, the percentage of INPH patients with depression was remarkably high even when compared to other dementias, ${ }^{27}$ more in line with the frequency of depression in patients with vascular dementia $(55 \%)^{58}$ and stroke ${ }^{59}$ than in patients with Alzheimer disease $(20 \%-30 \%){ }^{27}$

In patients with neurological impairment, the diagnosis of depression may be difficult because the symptomatology could be mistaken for symptoms of their neurological disease. ${ }^{27}$ If a patient with INPH is also suffering from depression, this will logically also affect the patient's cognitive state, the results of different cognitive tests, and his or her cognitive outcome after surgery. The cognitive symptoms of INPH are somewhat similar to the symptoms of depression, and when comparing the typical vascular depression and INPH the similarities are even more striking, with the typical frontal-subcortical executive dysfunction in both diagnoses. ${ }^{60-62}$ The same executive symptoms in 


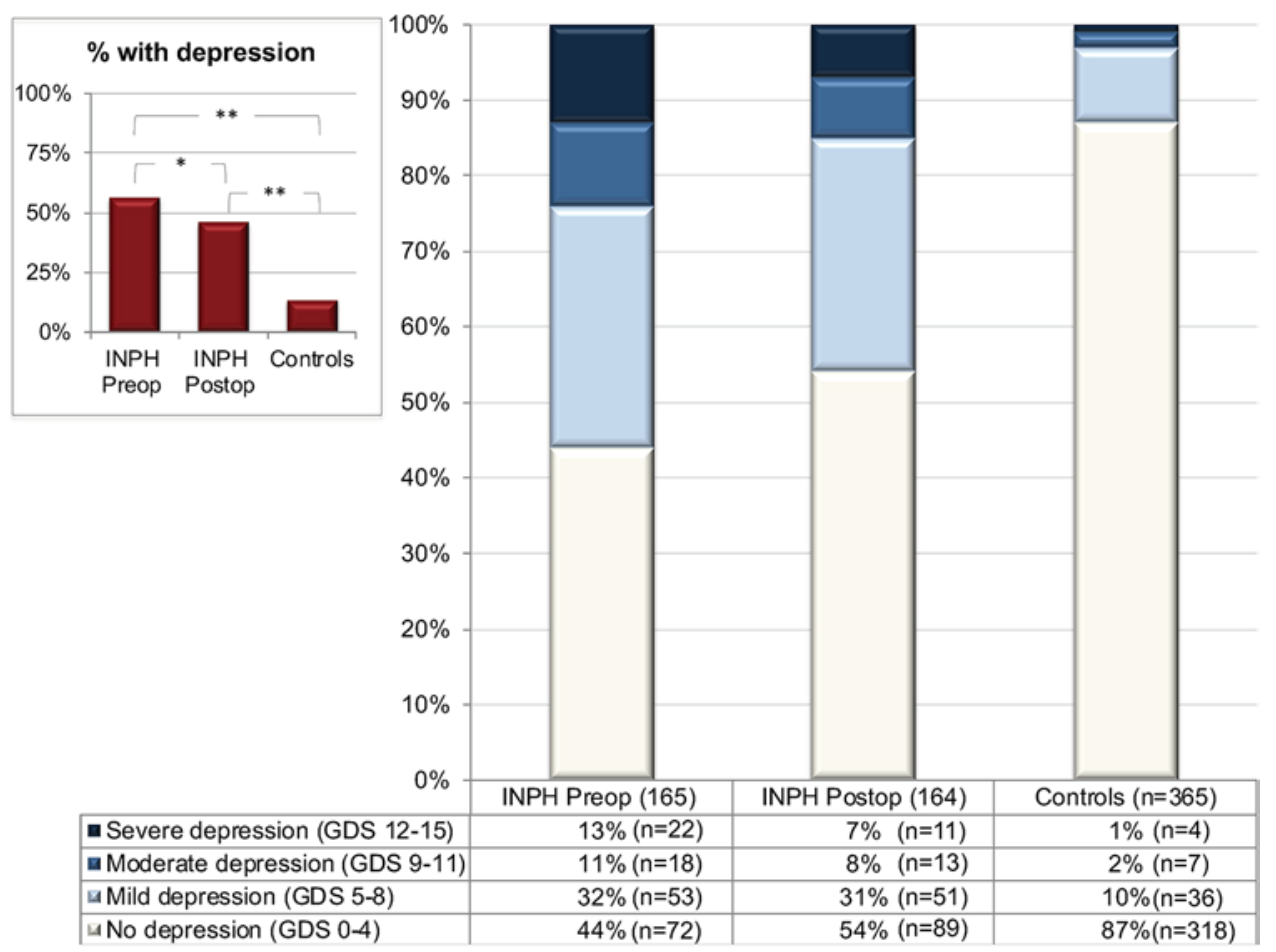

FIG. 1. Scoring on GDS-15 and severity of depression in INPH patients and controls. ${ }^{*} p<0.05,{ }^{* *} p<0.001$.

INPH are the ones least likely to improve after shunt treatment in INPH.$^{63}$ The INPH-CRasH study indicates that a lack of improvement in cognition after surgery may partly be explained by a coexisting, undiagnosed, and untreated depression. Few studies that investigate the neuropsychological profile of INPH report the frequency of depression. This means that a coexisting depression could act as a confounder, making the results of the neuropsychological tests unreliable.

\section{Suggestions for the Future}

The frequent comorbidity of depression among patients with INPH in combination with some similarities between the two diagnoses may mean that the diagnosis of INPH and subsequent treatment with shunt surgery may be delayed. However, the symptoms of INPH get worse over time, and a severe deterioration in function may not be possible to reverse. Thus, depression should be screened for as part of the clinical routine in INPH patients as well as in research. A depression scale should probably be incorporated when measuring the neuropsychological profile of INPH. If needed, adequate treatment for depression should probably be initiated as soon as possible. The effect of such interventions should be scientifically and clinically evaluated.

\section{Complications of Surgery}

\section{Minor AEs Are Common After Shunt Surgery}

The INPH-CRasH study reports high prevalences of mild and moderate AEs following shunt surgery for INPH, which in part conflict with previously published results. In certain aspects, the study can be considered unique in the field. In the INPH-CRasH study, definitive epilepsy was defined as a diagnosis of epilepsy received during in- or outpatient care, in combination with ongoing medication with antiepileptic drugs (AEDs). ${ }^{4}$ Both epilepsy and AEDs were overrepresented among patients with INPH compared to controls (epilepsy: $4.5 \%$ for patients vs $1.1 \%$ for controls, OR 4.3, 95\% CI 1.3-14.6, $\mathrm{p}=0.023$; AEDs: $14.8 \%$ vs $7.3 \%$, OR $0.5,95 \%$ CI $0.3-0.8, p=0.010$; Fig. 2 ). There was no difference before surgery (epilepsy: $2.3 \%$ vs $1.1 \%$, OR $2.1,95 \%$ CI $0.5-8.6, \mathrm{p}=0.280$; AEDs: $8.5 \%$ vs $5.4 \%$, OR $0.6,95 \%$ CI $0.3-1.2, p=0.235$ ). Insertion of a shunt should be considered the most probable cause for the overrepresentation of epilepsy among INPH patients compared to controls. This is in consideration of the vast difference in frequency of epilepsy diagnoses between the INPH and control populations after surgery, and the absence of a difference before surgery. Because single seizures and convulsions were not included, it is unlikely that the difference was caused by single surgically provoked seizures occurring immediately postoperatively. Because epilepsy is a disease with several severe implications in a person's daily life, it is an important AE to consider in preoperative evaluations. When our study was conducted, one previous study had been found reporting epilepsy after shunt surgery for INPH that was published almost 30 years ago and presented a prevalence of $9 \% .{ }^{64}$

Headache was also overrepresented among patients in whom shunts were placed for INPH compared to controls (36.1\% vs $11.6 \%$, OR $0.2,95 \%$ CI $0.2-0.4, p<0.001$; Fig. 2). Previous research regarding headache in patients with INPH focused mainly on postural headache induced by the shunt, and recent results show that it occurs in $8 \%$ of 


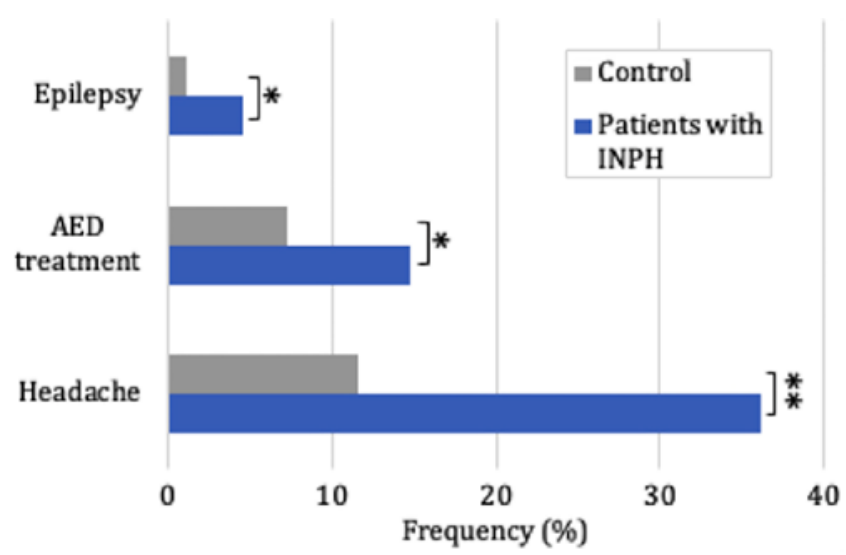

FIG. 2. Frequencies of headache, AED treatment, and epilepsy after shunt surgery for INPH and during the same time period for controls. " $p$ $<0.05,{ }^{* *} p<0.001$.

patients. ${ }^{16}$ In this study, $16 \%$ of shunt-treated patients had postural headache. Considering these results, headache must be considered a commonly occurring $\mathrm{AE}$ that patients should be informed about. Also, 8.5\% had experienced such severe headache that they had to visit a hospital, and the cause was intracranial hemorrhage in $27 \%$ of these cases. These findings stress the importance of clinicians considering hemorrhage in patients who receive shunts for INPH who present with rapidly evolving headache. Of the patients in whom shunts were place for INPH, 20\% experienced abdominal pain, persisting a mean of 21 months after surgery. Abdominal pain in an INPH population has rarely been investigated before, but previously published results indicate a frequency of approximately $1.5 \%$ after shunt surgery. ${ }^{65,66}$ The frequency of chronic or persistent abdominal pain in the general population ranges between $1.2 \%$ (the general population of the United States) ${ }^{67}$ and $6 \%$ (older people in low- and middle-income countries). Hence, the high frequency of abdominal discomfort experienced by patients in this study was probably caused by direct irritation by the shunt on intraabdominal structures.

\section{Suggestions for the Future}

In addition to the commonly investigated serious AEs, such as shunt infections and subdural hemorrhages, mild and moderate AEs such as epilepsy, headache, and abdominal pain should be further evaluated in future research and included in postoperative clinical follow-ups.

\section{Quality of Life}

QOL Is an Important Outcome Measure After Surgery

A main goal of surgery should be to improve QOL, and in recent years dementia research has supported the importance of measuring QOL to evaluate the effect of a treatment in elderly individuals. ${ }^{68}$ However, appropriate measurements of QOL are often disregarded when evaluating the effect of shunt surgery in INPH. In the INPHCRaSH study, up to $75 \%$ of the INPH patients reported improvements in QOL after shunting (72\% reported improvement in the general measure EQ-VAS and $75 \%$ reported improvement in the 5-item EQ-5D-5L index). This improvement was long lasting (mean length of follow-up 21 months). Also, the means ( \pm SD) of both QOL measurements were higher after shunt surgery (EQ-VAS score: preoperative, $45 \pm 24$; postoperative, $67 \pm 21$; mean difference $22 \pm 30$ [p < 0.001]; EQ-5D-5L index: preoperative, $0.45 \pm 0.31$; postoperative, $0.62 \pm 0.27$; mean difference $0.17 \pm 0.33$ [p < 0.001]) (Fig. 3). A long-lasting improvement of QOL after shunting is in accordance with what has previously been suggested in short-term studies and studies assessing indirect QOL.44,69-72

When compared to controls, INPH patients had lower QOL both before and after surgery ( $\mathrm{p}<0.001$ for both QOL measurements, mean [ $\pm \mathrm{SD}] \mathrm{EQ}-\mathrm{VAS}$ score for controls: $79 \pm 17$; mean $[ \pm \mathrm{SD}] \mathrm{EQ}-5 \mathrm{D}-5 \mathrm{~L}$ index for controls: $0.80 \pm 0.19$ ), and one of the most important predictors for low QOL in shunt-treated INPH patients was a coexisting depression $(\mathrm{p}<0.001)$. This was in accordance with what has previously been reported in INPH patients before surgery compared to the elderly population. ${ }^{73}$ When compared to other dementias, INPH seems to have a higher impact on QOL than Alzheimer disease ${ }^{74}$ and an equal impact on QOL to Parkinson disease..$^{75}$

Another finding in the INPH-CRasH study was that it seems to be the severity of the symptoms $(p<0.001)$ rather than the outcome of surgery $(p=0.02-0.065)$ that influences QOL in shunt-treated INPH patients. ${ }^{3}$ Because the symptoms of INPH gets worse over time, these results underscore the importance of correct and timely diagnosis and treatment. ${ }^{8,9}$

\section{Suggestions for the Future}

Measures regarding QOL need to be incorporated in the evaluation of surgery, both in clinical care and in research. A prospective analysis of depression and QOL should probably be conducted.

\section{Conclusions} lows:

Conclusions from the INPH-CRasHc study were as fol-

1. Patients with INPH have more VRFs than the normal elderly population. The most important VRFs in INPH are diabetes, hyperlipidemia, obesity, psychosocial factors, physical inactivity, and hypertension. The population attributable risk percent for a model containing all of the VRFs associated with INPH was $24 \%$. This suggests that vascular disease, particularly cerebral smallvessel disease, may be one underlying pathophysiological cause of INPH. In the clinical care of INPH patients, VRFs should be screened for, and treatment for coexisting risk factors should be optimized. ${ }^{1}$

2. Symptoms of depression are overrepresented in INPH patients compared with the normal elderly population, despite treatment with a shunt. Screening for depression should be included in the clinical routine and in neuropsychological testing of INPH patients. ${ }^{2}$

3. QOL remains improved in shunt-treated INPH patients at a mean follow-up time of 21 months. However, shunttreated INPH patients still have a lower QOL than the normal elderly population. The main predictors for low QOL among shunt-treated INPH patients are a coexist- 


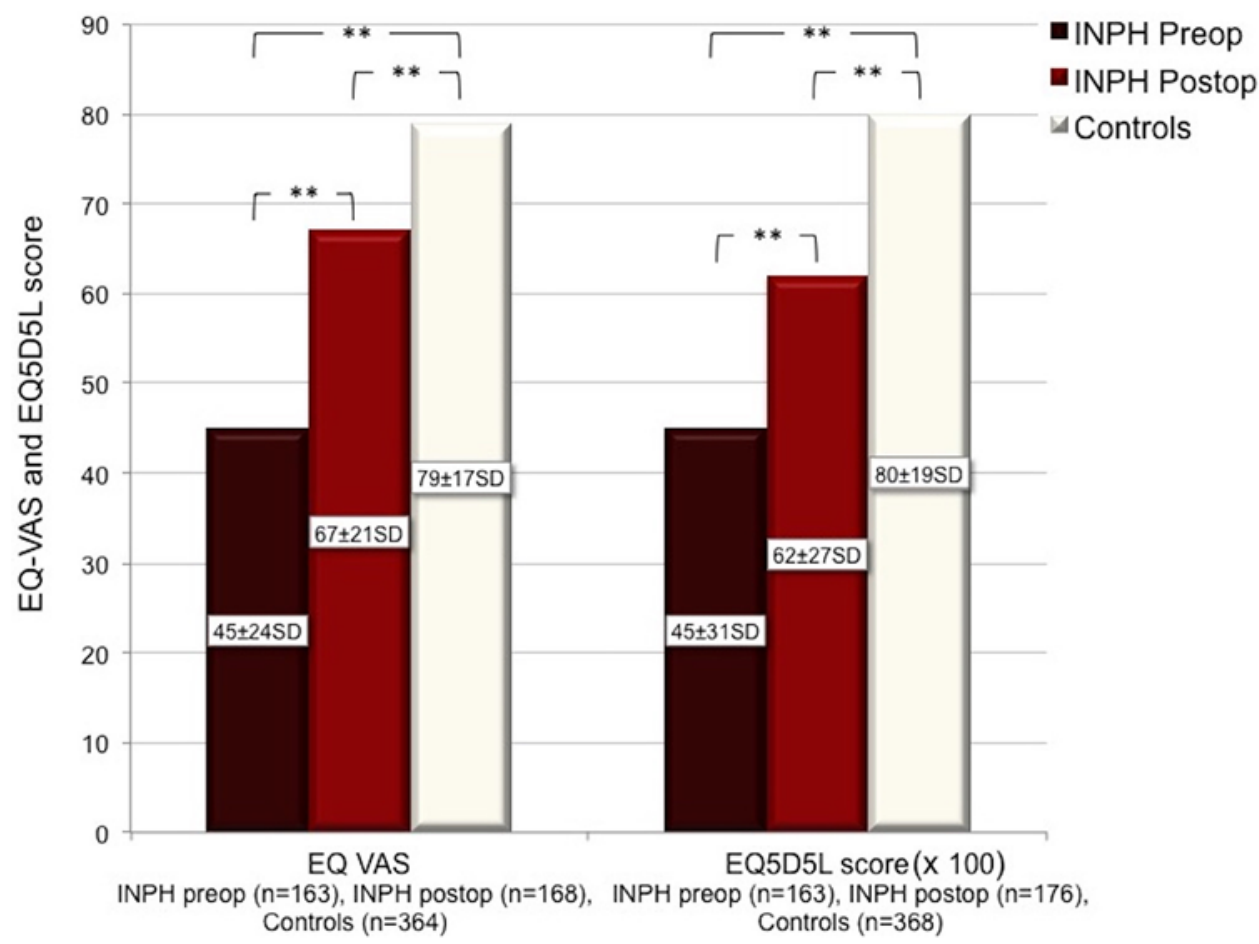

FIG. 3. The mean values for QOL according to EQ-VAS and EQ-5D-5L in INPH patients and controls. ${ }^{* *} p<0.001$.

ing depression and severity of gait disturbance, not outcome of surgery. ${ }^{3}$

4. To prove a proper causal relationship between INPH and VRFs/vascular disease, a large longitudinal populationbased study should be conducted with a design similar to the INPH-CRasH study, investigating if VRFs and CVD exist before the diagnosis of INPH. ${ }^{5}$

5. In clinical follow-up visits after shunt surgery in INPH, mild and moderate AEs such as epilepsy, headache, and abdominal pain should be assessed. When developing new methods for shunt placement, mild and moderate AEs should be considered. ${ }^{4}$

\section{Acknowledgments}

We acknowledge Kristin Nyman, research nurse, for valuable help. This study was supported by The Swedish Research Council, a regional agreement between Umeå University and Västerbotten County Council, and the research fund for clinical neuroscience at Umeå University Hospital. These are all governmental or institutional nonprofit organizations without any economic interest or practical involvement in the study.

\section{References}

1. Israelsson H, Carlberg B, Wikkelsö C, et al. Vascular risk factors in INPH: a prospective case-control study (the INPHCRasH study). Neurology. 2017;88(6):577-585.

2. Israelsson H, Allard P, Eklund A, Malm J. Symptoms of depression are common in patients with idiopathic normal pressure hydrocephalus: the INPH-CRasH study. Neurosurgery. 2016;78(2):161-168.

3. Israelsson H, Eklund A, Malm J. Cerebrospinal fluid shunting improves long-term quality of life in idiopathic normal pressure hydrocephalus. Neurosurgery. 2020;86(4):574-582.
4. Larsson J, Israelsson H, Eklund A, Malm J. Epilepsy, headache, and abdominal pain after shunt surgery for idiopathic normal pressure hydrocephalus: the INPH-CRasH study. $J$ Neurosurg. 2018;128(6):1674-1683.

5. Israelsson H. Comorbidity and vascular risk factors associated with idiopathic normal pressure hydrocephalus: the INPH-CRasH study [dissertation]. Umeå University; 2016. Accessed August 6, 2020. http://umu.diva-portal.org/smash/ get/diva2:927077/FULLTEXT01.pdf

6. Relkin N, Marmarou A, Klinge P, et al. Diagnosing idiopathic normal-pressure hydrocephalus. Neurosurgery. 2005; 57(3)(suppl):S4-S16, ii-v.

7. Williams MA, Malm J. Diagnosis and treatment of idiopathic normal pressure hydrocephalus. Continuum (Minneap Minn). 2016;22(2 Dementia):579-599.

8. Andrén K, Wikkelsø C, Tisell M, Hellström P. Natural course of idiopathic normal pressure hydrocephalus. J Neurol Neurosurg Psychiatry. 2014;85(7):806-810.

9. Kazui H, Miyajima M, Mori E, Ishikawa M. Lumboperitoneal shunt surgery for idiopathic normal pressure hydrocephalus (SINPHONI-2): an open-label randomised trial. Lancet Neurol. 2015;14(6):585-594.

10. Klinge P, Hellström P, Tans J, Wikkels $\varnothing$ C. One-year outcome in the European multicentre study on iNPH. Acta Neurol Scand. 2012;126(3):145-153.

11. Kahlon B, Sjunnesson J, Rehncrona S. Long-term outcome in patients with suspected normal pressure hydrocephalus. Neurosurgery. 2007;60(2):327-332.

12. Toma AK, Papadopoulos MC, Stapleton S, et al. Systematic review of the outcome of shunt surgery in idiopathic normalpressure hydrocephalus. Acta Neurochir (Wien). 2013; 155(10):1977-1980.

13. Duinkerke A, Williams MA, Rigamonti D, Hillis AE. Cognitive recovery in idiopathic normal pressure hydrocephalus after shunt. Cogn Behav Neurol. 2004;17(3):179-184.

14. Thomas G, McGirt MJ, Woodworth G, et al. Baseline neuropsychological profile and cognitive response to cerebrospinal 
fluid shunting for idiopathic normal pressure hydrocephalus. Dement Geriatr Cogn Disord. 2005;20(2-3):163-168.

15. Sundström N, Malm J, Laurell K, et al. Incidence and outcome of surgery for adult hydrocephalus patients in Sweden. Br J Neurosurg. 2017;31(1):21-27.

16. Hashimoto M, Ishikawa M, Mori E, Kuwana N. Diagnosis of idiopathic normal pressure hydrocephalus is supported by MRI-based scheme: a prospective cohort study. Cerebrospinal Fluid Res. 2010;7:18.

17. Malm J, Graff-Radford NR, Ishikawa M, et al. Influence of comorbidities in idiopathic normal pressure hydrocephalus research and clinical care. A report of the ISHCSF task force on comorbidities in INPH. Fluids Barriers CNS. 2013;10(1): 22.

18. Bradley WG Jr, Whittemore AR, Watanabe AS, et al. Association of deep white matter infarction with chronic communicating hydrocephalus: implications regarding the possible origin of normal-pressure hydrocephalus. AJNR Am J Neuroradiol. 1991;12(1):31-39.

19. Momjian S, Owler BK, Czosnyka Z, et al. Pattern of white matter regional cerebral blood flow and autoregulation in normal pressure hydrocephalus. Brain. 2004;127(Pt 5):965972.

20. Bateman GA. Pulse wave encephalopathy: a spectrum hypothesis incorporating Alzheimer's disease, vascular dementia and normal pressure hydrocephalus. Med Hypotheses. 2004;62(2):182-187.

21. Boon AJ, Tans JT, Delwel EJ, et al. Dutch Normal-Pressure Hydrocephalus Study: the role of cerebrovascular disease. $J$ Neurosurg. 1999;90(2):221-226.

22. Perk J, De Backer G, Gohlke H, et al. European guidelines on cardiovascular disease prevention in clinical practice (version 2012): the fifth joint task force of the European Society of Cardiology and other societies on cardiovascular disease prevention in clinical practice (constituted by representatives of nine societies and by invited experts). Int J Behav Med. 2012; 19(4):403-488.

23. Jacobs L. Diabetes mellitus in normal pressure hydrocephalus. J Neurol Neurosurg Psychiatry. 1977;40(4):331-335.

24. Graff-Radford NR, Godersky JC. Idiopathic normal pressure hydrocephalus and systemic hypertension. Neurology. 1987; 37(5):868-871.

25. Casmiro M, D'Alessandro R, Cacciatore FM, et al. Risk factors for the syndrome of ventricular enlargement with gait apraxia (idiopathic normal pressure hydrocephalus): a casecontrol study. J Neurol Neurosurg Psychiatry. 1989;52(7): 847-852.

26. Krauss JK, Regel JP, Vach W, et al. Vascular risk factors and arteriosclerotic disease in idiopathic normal-pressure hydrocephalus of the elderly. Stroke. 1996;27(1):24-29.

27. Enache D, Winblad B, Aarsland D. Depression in dementia: epidemiology, mechanisms, and treatment. Curr Opin Psychiatry. 2011;24(6):461-472.

28. Djernes JK. Prevalence and predictors of depression in populations of elderly: a review. Acta Psychiatr Scand. 2006; 113(5):372-387.

29. Yusuf S, Hawken S, Ounpuu S, et al. Effect of potentially modifiable risk factors associated with myocardial infarction in 52 countries (the INTERHEART study): case-control study. Lancet. 2004;364(9438):937-952.

30. O'Donnell MJ, Xavier D, Liu L, et al. Risk factors for ischaemic and intracerebral haemorrhagic stroke in 22 countries (the INTERSTROKE study): a case-control study. Lancet. 2010;376(9735):112-123.

31. Sheikh JI, Yesavage JA. Geriatric Depression Scale (GDS): recent evidence and development of a shorter version. Clin Gerontol. 1986;5(1-2):165-173.

32. EuroQol Group. EuroQol-a new facility for the measurement of health-related quality of life. Health Policy. 1990; 16(3):199-208.
33. Hellström K, Lindmark B. Fear of falling in patients with stroke: a reliability study. Clin Rehabil. 1999;13(6):509-517.

34. Larsson J, Israelsson H, Eklund A, Malm J. Falls and fear of falling are common in idiopathic normal pressure hydrocephalus. In: Hydrocephalus 2017, the Ninth Annual Meeting of the International Society for Hydrocephalus and CSF disorders (Hydrocephalus Society). Fluids Barriers CNS. 2018; 15(Suppl 1):4.

35. Agren-Wilsson A, Roslin M, Eklund A, et al. Intracerebral microdialysis and CSF hydrodynamics in idiopathic adult hydrocephalus syndrome. J Neurol Neurosurg Psychiatry. 2003;74(2):217-221.

36. Gorelick PB, Scuteri A, Black SE, et al. Vascular contributions to cognitive impairment and dementia: a statement for healthcare professionals from the American Heart Association/American Stroke Association. Stroke. 2011;42(9): 2672-2713.

37. Eide PK, Pripp AH. Increased prevalence of cardiovascular disease in idiopathic normal pressure hydrocephalus patients compared to a population-based cohort from the HUNT3 survey. Fluids Barriers CNS. 2014;11:19.

38. Jaraj D, Agerskov S, Rabiei K, et al. Vascular factors in suspected normal pressure hydrocephalus: a population-based study. Neurology. 2016;86(7):592-599.

39. Hudson M, Nowak C, Garling RJ, Harris C. Comorbidity of diabetes mellitus in idiopathic normal pressure hydrocephalus: a systematic literature review. Fluids Barriers CNS. 2019;16(1):5.

40. Ghaffari-Rafi A, Gorenflo R, Hu H, et al. Role of psychiatric, cardiovascular, socioeconomic, and demographic risk factors on idiopathic normal pressure hydrocephalus: a retrospective case-control study. Clin Neurol Neurosurg. 2020;193:105836.

41. Deckers K, van Boxtel MP, Schiepers OJ, et al. Target risk factors for dementia prevention: a systematic review and Delphi consensus study on the evidence from observational studies. Int J Geriatr Psychiatry. 2015;30(3):234-246.

42. Malm J, Kristensen B, Karlsson T, et al. The predictive value of cerebrospinal fluid dynamic tests in patients with th idiopathic adult hydrocephalus syndrome. Arch Neurol. 1995; 52(8):783-789.

43. Krauss JK, Droste DW, Vach W, et al. Cerebrospinal fluid shunting in idiopathic normal-pressure hydrocephalus of the elderly: effect of periventricular and deep white matter lesions. Neurosurgery. 1996;39(2):292-300.

44. Spagnoli D, Innocenti L, Bello L, et al. Impact of cerebrovascular disease on the surgical treatment of idiopathic normal pressure hydrocephalus. Neurosurgery. 2006;59(3):545-552.

45. Klinge P, Marmarou A, Bergsneider M, et al. Outcome of shunting in idiopathic normal-pressure hydrocephalus and the value of outcome assessment in shunted patients. Neurosurgery. 2005;57(3)(suppl):S40-S52, ii-v.

46. Bådagård $H$, Braun $M$, Nilsson $D$, et al. Negative predictors of shunt surgery outcome in normal pressure hydrocephalus. Acta Neurol Scand. 2020;141(3):219-225.

47. Andrén K, Wikkelsö C, Sundström N, et al. Long-term effects of complications and vascular comorbidity in idiopathic normal pressure hydrocephalus: a quality registry study. $J$ Neurol. 2018;265(1):178-186.

48. Leinonen V, Koivisto AM, Alafuzoff I, et al. Cortical brain biopsy in long-term prognostication of 468 patients with possible normal pressure hydrocephalus. Neurodegener Dis. 2012;10(1-4):166-169.

49. Andrén K, Wikkelsø C, Sundström N, et al. Survival in treated idiopathic normal pressure hydrocephalus. J Neurol. 2020;267(3):640-648

50. Baezner H, Blahak C, Poggesi A, et al. Association of gait and balance disorders with age-related white matter changes: the LADIS study. Neurology. 2008;70(12):935-942.

51. Johansson E, Ambarki K, Birgander R, et al. Cerebral micro- 
bleeds in idiopathic normal pressure hydrocephalus. Fluids Barriers CNS. 2016;13(1):4.

52. Tisell M, Tullberg M, Hellström P, et al. Shunt surgery in patients with hydrocephalus and white matter changes. $J$ Neurosurg. 2011;114(5):1432-1438.

53. Bech-Azeddine R, Høgh P, Juhler M, et al. Idiopathic normal-pressure hydrocephalus: clinical comorbidity correlated with cerebral biopsy findings and outcome of cerebrospinal fluid shunting. J Neurol Neurosurg Psychiatry. 2007;78(2): 157-161.

54. Manniche C, Simonsen AH, Hasselbalch SG, et al. Cerebrospinal fluid biomarkers to differentiate idiopathic normal pressure hydrocephalus from subcortical ischemic vascular disease. J Alzheimers Dis. 2020;75(3):937-947.

55. Sakakibara R, Uchida Y, Ishii K, et al. Correlation of right frontal hypoperfusion and urinary dysfunction in iNPH: a SPECT study. Neurourol Urodyn. 2012;31(1):50-55.

56. Tullberg M, Hellström P, Piechnik SK, et al. Impaired wakefulness is associated with reduced anterior cingulate CBF in patients with normal pressure hydrocephalus. Acta Neurol Scand. 2004;110(5):322-330.

57. Chang CC, Asada H, Mimura T, Suzuki S. A prospective study of cerebral blood flow and cerebrovascular reactivity to acetazolamide in 162 patients with idiopathic normalpressure hydrocephalus. J Neurosurg. 2009;111(3):610-617.

58. Moretti R, Torre P, Antonello RM, Cazzato G. Behavioral alterations and vascular dementia. Neurologist. 2006;12(1): 43-47.

59. Hornsten C, Molander L, Gustafson Y. The prevalence of stroke and the association between stroke and depression among a very old population. Arch Gerontol Geriatr. 2012; 55(3):555-559.

60. Taylor WD, Aizenstein HJ, Alexopoulos GS. The vascular depression hypothesis: mechanisms linking vascular disease with depression. Mol Psychiatry. 2013;18(9):963-974.

61. Alexopoulos GS. Depression in the elderly. Lancet. 2005; 365(9475):1961-1970.

62. Hellström P, Klinge P, Tans J, Wikkelsø C. The neuropsychology of iNPH: findings and evaluation of tests in the European multicentre study. Clin Neurol Neurosurg. 2012; 114(2):130-134.

63. Peterson KA, Savulich G, Jackson D, et al. The effect of shunt surgery on neuropsychological performance in normal pressure hydrocephalus: a systematic review and meta-analysis. J Neurol. 2016;263(8):1669-1677.

64. Larsson A, Wikkelsö C, Bilting M, Stephensen H. Clinical parameters in 74 consecutive patients shunt operated for normal pressure hydrocephalus. Acta Neurol Scand. 1991;84(6): 475-482.

65. Eggenberger ER, Miller NR, Vitale S. Lumboperitoneal shunt for the treatment of pseudotumor cerebri. Neurology. 1996;46(6):1524-1530.

66. Schucht P, Banz V, Trochsler M, et al. Laparoscopically assisted ventriculoperitoneal shunt placement: a prospective randomized controlled trial. J Neurosurg. 2015;122(5): $1058-1067$.

67. Hardt J, Jacobsen C, Goldberg J, et al. Prevalence of chronic pain in a representative sample in the United States. Pain Med. 2008;9(7):803-812.
68. Haywood KL, Garratt AM, Fitzpatrick R. Quality of life in older people: a structured review of generic self-assessed health instruments. Qual Life Res. 2005;14(7):1651-1668.

69. Katzen H, Ravdin LD, Assuras S, et al. Postshunt cognitive and functional improvement in idiopathic normal pressure hydrocephalus. Neurosurgery. 2011;68(2):416-419.

70. Lemcke J, Meier U, Müller C, et al. Safety and efficacy of gravitational shunt valves in patients with idiopathic normal pressure hydrocephalus: a pragmatic, randomised, open label, multicentre trial (SVASONA). J Neurol Neurosurg Psychiatry. 2013;84(8):850-857.

71. Petersen J, Hellström P, Wikkels $\varnothing$ C, Lundgren-Nilsson A. Improvement in social function and health-related quality of life after shunt surgery for idiopathic normal-pressure hydrocephalus. J Neurosurg. 2014;121(4):776-784.

72. Cage TA, Auguste KI, Wrensch M, et al. Self-reported functional outcome after surgical intervention in patients with idiopathic normal pressure hydrocephalus. J Clin Neurosci. 2011;18(5):649-654.

73. Junkkari A, Sintonen H, Nerg O, et al. Health-related quality of life in patients with idiopathic normal pressure hydrocephalus. Eur J Neurol. 2015;22(10):1391-1399.

74. Jönsson L, Andreasen N, Kilander L, et al. Patient- and proxy-reported utility in Alzheimer disease using the EuroQoL. Alzheimer Dis Assoc Disord. 2006;20(1):49-55.

75. Alvarado-Bolaños A, Cervantes-Arriaga A, Rodríguez-Violante $\mathrm{M}$, et al. Convergent validation of EQ-5D-5L in patients with Parkinson's disease. J Neurol Sci. 2015;358(1-2):53-57.

\section{Disclosures}

The authors report no conflict of interest concerning the materials or methods used in this study or the findings specified in this paper.

\section{Author Contributions}

Conception and design: Israelsson, Eklund, Malm. Acquisition of data: Israelsson. Analysis and interpretation of data: all authors. Drafting the article: Israelsson. Critically revising the article: all authors. Reviewed submitted version of manuscript: all authors. Approved the final version of the manuscript on behalf of all authors: Israelsson. Statistical analysis: all authors. Study supervision: Israelsson, Eklund, Malm.

\section{Supplemental Information}

\section{Previous Presentations}

Portions of this work were presented in Dr. Israelsson's dissertation: Israelsson H. Comorbidity and vascular risk factors associated with idiopathic normal pressure hydrocephalus: the INPHCRasH study. Umeå University; 2016. Accessed August 6, 2020. http://umu.diva-portal.org/smash/get/diva2:927077/FULLTEXT01. pdf.

\section{Correspondence}

Hanna Israelsson: Umeå University, Umeå, Sweden. hanna. israelsson@umu.se. 